\title{
Gesetzliche Grenzen der Anti-Werbung für ärztliche Leistungen und Ansehen
}

\section{Einführung}

$\nabla$

Der britische Soziologe und Politiker Michael Young prägte mit seiner satirischen Schrift „The Rise of Meritocracy“ den Terminus Meritokratie, der nunmehr Einzug in das Internet gehalten hat. In der Meritokratie geht es um die Vergabe von Ansehen und Finanzen via Leistung. Über Wertschätzung und damit Praxiserfolg entscheidet die Internet-Community. Sie vergibt Punkte, Sterne oder andere Symbole, in Anlehnung an das System der Schulnoten. Ferner machen sich Patienten mit Erfahrungsberichten und die Medien die Mühe, die Leistung von Ärzten auszuweisen. Durch das Internet können dabei falsche Eindrücke aufgrund mangelnder Urteilskraft der Beitragsverfasser entstehen. Diese sind nicht nur psychologisch, sondern auch medial widerlegungsresistenter als ihre positiven Pendants. Diese Anti-Werbung hat einen rechtlichen Rahmen, der nachfolgend dargestellt wird. Im Fokus stehen die zivilrechtlichen Haftungsansprüche aufgrund von Verletzungen der Persönlichkeitsrechte des Arztes. Schlussendlich werden die spiegelbildlichen strafrechtlichen Implikationen diskutiert, deren Täter und Opfer zugleich der Arzt sein kann.

\section{Arzt-Bewertungsportal}

$\nabla$

\section{Hintergrund}

Hintergrund des zivilrechtlichen Beschlusses des Landgerichts Nürnberg-Fürth [1] ist, dass in einem Online-Arzt-Bewertungsportal ein Nutzer die zahnärztliche Leistung wie folgt beschrieb und bewertete:

„Dieser Arzt arbeitet leider nur nach Quantität als auf Qualität zu setzen und ist ganz schnell mit Kronen einsetzen, obwohl es vielleicht noch gar nicht nötig wäre. Hatte durch Unfall einige Kronen bekommen, die leider für ihren Preis von mehreren Tausend EUR sehr schlecht im Mund eingearbeitet wurden, so dass ich seit dem immer Zahnfleischbluten habe und anfangs öfters die eine Krone verloren habe bis ich zu einem anderen Arzt ging. Die Farbe der Keramik passt mit der Farbe meiner Zähne nicht überein, Implantate sind gegenüber meinen anderen Zähnen zu groß usw. könnte hier jetzt noch mehr aufzählen was ich mit diesem Arzt erlebt habe, aber diese würde das ganze hier nur sprengen. Wenn ihr eure Zähne behalten wollt dann geht woanders hin ..."
Auf anwaltliche Aufforderung, die Bewertung zu löschen, nahm der Portal-Betreiber Rücksprache mit dem Nutzer. Er erhielt vom Nutzer die E-Mail „Hallo, ja der Sachverhalt hat sich so zugetragen! Mit freundlichen Grüßen“ und löschte den Satz „Wenn ihr eure Zähne behalten wollt dann geht woanders hin.....

\section{Erwågungen}

Das Landgericht gab der beantragten einstweiligen Verfügung des Arztes statt, indem es dem Betreiber des Arzt-Bewertungsportals untersagte, die negativen Bewertungen zu behaupten, behaupten zu lassen oder hierfür die Plattform zur Verfügung zu stellen.

Der Zahnarzt konnte das Unterlassungsbegehren nicht auf eine Kreditgefährdung gemäß §824 BGB stützen. Er legte nicht dar, dass sich infolge der gerügten Bewertung bereits Patienten bei ihm abgemeldet oder von einer (Neu-)Anmeldung abgesehen hätten. Der Unterlassungsanspruch des Zahnarztes ergab sich aus seinem allgemeinen Persönlichkeitsrecht und dem Recht am eingerichteten und ausgeübten Gewerbebetrieb der Zahnarztpraxis als „sonstige Rechte“ im Sinne von §823 Abs.1 BGB i.V.m. $§ 1004$ Abs.1 S.2 BGB analog. Die Rechtsauffassungen gehen hinsichtlich der Frage auseinander, ob ein Arzt nicht schon deswegen daran gehindert ist, sich auf das Recht am eingerichteten und ausgeübten Gewerbebetrieb zu berufen, weil ein Arzt mit anderen Ärzten im Wettbewerb steht (so wohl OLG Frankfurt [2]). Das Landgericht folgte nicht der zuvor ergangenen Rechtsprechung des OLG Frankfurt aufgrund einer Folgenbetrachtung. Das auch den Ärzten und Zahnärzten zustehende Recht am eingerichteten und ausgeübten Gewerbebetrieb liefe nämlich ansonsten in Bezug auf ehrbeeinträchtigende, damit letztlich auch geschäftsschädigende unzutreffende Tatsachenbehauptungen leer. Denn nahezu alle Gewerbetreibenden stünden in Wettbewerb zu Konkurrenten am Markt. Für die Störerhaftung des Portal-Betreibers bewog das Landgericht, dass der Portalinhaber die Webseite betreibt, dabei den Speicherplatz für die von den Nutzern verfassten Beiträge bzw. Bewertungen bereitstellt und den Abruf dieser Webseite über das Internet ermöglicht. Denn damit wird willentlich und adäquat kausal zur Verbreitung von Äußerungen beigetragen, die das allgemeine Persönlichkeitsrecht Dritter beeinträchtigen können. Die haftungsrechtliche Verantwortlichkeit folgte nicht aus einer sogenannten Intellektuellenverbreitung, denn dafür fehlt es an einer gedanklichen Beziehung zu dem Inhalt des Nutzers. Sie ergab
T. Oehler ${ }^{1}$

Arztrecht

Schlüsselwörter

Meritokratie

Online-Arzt-

Bewertungsportal

Erfahrungsbericht

Internet-Blog

Keywords

meritocracy

online doctor review site

- experience report

internet blog

Institut

Advokaturbüro Oehler,

Osnabrück

eingereicht 13.03.2013

akzeptiert 16.05.2013

Bibliografie

DOI 10.1055/s-0033-1343237

Dtsch Med Wochenschr 2013;

138: 1373-1376 - (c) Georg

Thieme Verlag KG · Stuttgart .

New York · ISSN 0012-0472

Korrespondenzadresse Tim Oehler, Rechtsanwalt Advokaturbüro Oehler Berningstr. 1a 49090 Osnabrück eMail advokatur@gmx.de 
sich aus der technischen Verbreitung und einer Abwägung des Rechts des Zahnarztes auf Schutz seiner Persönlichkeit gegenüber der Meinungsfreiheit bzw. Medienfreiheit. Von entscheidender Bedeutung war, dass die Äußerungen nicht als Meinung, sondern als Tatsachenbehauptung eingestuft wurden.

Die notenmäßige Beurteilung konnte nicht schützend vor den Fließtext gestellt werden, der die Zensierung begründen sollte. In den Vordergrund traten nämlich die dem Beweis zugänglichen Tatsachenbehauptungen:

- der Zahnarzt arbeite „nur nach Quantität als auf Qualität zu setzen“;

- er sei „ganz schnell mit Kronen einsetzen, obwohl es vielleicht noch gar nicht nötig wäre“;

- er habe Kronen eingesetzt, die sehr schlecht im Mund eingearbeitet wurden, sodass es beim Patienten zu Zahnfleischbluten komme und die Kronen herausfielen;

- er setze Keramik ein, deren Farbe nicht zur Farbe der Zähne der Patienten passe und/oder er setze zu große Implantate ein.

Diese Äußerungen können bezogen auf eine konkrete zahnärztliche Behandlung im Rahmen einer Begutachtung durch einen zahnärztlichen Sachverständigen geklärt werden. Die Fragen, die der Sachverständige im Rahmen einer Begutachtung, z.B. auch im Rahmen eines zivilrechtlichen Arzthaftungsprozesses $\mathrm{zu}$ beantworten hätte, wären darauf gerichtet, ob die Behandlung durch den Zahnarzt ohne ärztlichen Kunstfehler erfolgte, also indiziert war, fehlerfrei durchgeführt und auch ordnungsgemäß abgerechnet wurde. Käme der Sachverständige zu dem für den Zahnarzt günstigen Ergebnis, dass ein Behandlungsfehler nicht vorliegt, wäre damit auch geklärt, dass der Zahnarzt anlässlich der der Bewertung zugrunde liegenden Behandlung nicht sein wirtschaftliches Interesse in den Vordergrund gestellt hat. Seine konkrete ärztliche Behandlung entsprach dem anerkannten und gesicherten Standard der ärztlichen Wissenschaft - er ließ also nach den Erkenntnissen der ärztlichen Wissenschaft unter den jeweiligen Umständen die objektiv erforderliche Sorgfalt nicht außer Acht. Gemäß dem Urteil verletzte der Portalbetreiber aber seine Prüfpflichten. Er durfte sich nicht damit zufrieden geben, die Beanstandung des Zahnarztes an den für den Beitrag Ver- antwortlichen zur Stellungnahme weiterzuleiten und das lapidare Feedback zu empfangen „Hallo, ja der Sachverhalt hat sich so zugetragen! Mit freundlichen Grüßen“. Dies wäre nur dann rechtskonform, wenn der Verfasser des Beitrags die Beanstandung durch den Zahnarzt substantiiert in Abrede gestellt hätte und sich daraus berechtigte Zweifel an der Darstellung des Zahnarztes ergeben hätten. Anders verhielt es sich hier, wonach der Zahnarzt seinerseits substantiiert mitteilte, worauf sich seine Beanstandung gründete. Dies hätte Anlass für den Portalbetreiber sein müssen, einen geeigneten Nachweis für die Durchführung der Behandlung (z.B. unkenntlich gemachte Honorarrechnung des Zahnarztes) vom Ersteller des Beitrags zu verlangen.

\section{Google-Erfahrungsbericht}

Ärzte können ihre Arztpraxis im Internet nicht nur über die eigene Webseite, sondern auch über „Google Places“ präsentieren. Auf dieser Plattform können Erfahrungsberichte zur Arztpraxis und den Behandelnden (durch z.B. Patienten) eingestellt werden.

\section{Hintergrund}

Hintergrund des zur Veröffentlichung eingestellten Zivilurteils des Landgerichts Berlin [3] war, dass ein Nutzer von der Möglichkeit Gebrauch machte, eine persönliche Stellungnahme zu angezeigten Suchergebnissen (Erfahrungsbericht) bei einem Internet-Suchdienstanbieter abzugeben. Unter einem Pseudonym wurde die Darstellung über das vermeintliche Erleben einer ärztlichen Behandlung abgesetzt:

„Vorsicht!!!!!!!!!!!! der Fuscher!!!! schlimmer kann man einen Menschen nicht verunstalten: seit dieser ,Behandlung' kann ich nicht mehr anziehen, was ich will, ich muss genau überlegen womit ich was abdecken kann. Meine Arme, Mein Po - alles mit Dellen überseht und hängt unvorstellbar hässlich $a b$. Was ich schon investiert habe in Korrekturoperationen - > nichts hilft mehr! Seid vorsichtig! Seid gewarnt!!! Er ist furchtbar!“

Der Arzt forderte zur Entfernung des Eintrags auf und beantragte sodann eine einstweilige Verfügung. Nachdem diese abgelehnt wurde, zog er via Beschwerde weiter, und zwar mit Erfolg. Zwischen- zeitlich hatte der Arzt auf den „verleumderisch[en] und beleidigend[en]“ Inhalt hingewiesen.

\section{Erwågungen}

Das Landgericht Berlin folgte dem Antrag des Arztes - gestützt auf $\S 1004$ Abs.1 S.2 BGB analog - gegenüber dem Hostprovider, dass dieser es unterlassen sollte, wörtlich oder sinngemäß die oben zitierte Behauptung zu verbreiten. Auf den ersten Blick ist Google kein typischer ArztBewertungs-Portalbetreiber. Die Grundsätze der Störerhaftung beanspruchen trotzdem auch hier Geltung. Ein Hostprovider ist grundsätzlich nicht verpflichtet, die von den Nutzern in das Internet gestellten Beiträge vor der Veröffentlichung auf eventuelle Rechtsverletzungen $\mathrm{zu}$ überprüfen. Er haftet dann aber, sobald er Kenntnis von der Rechtsverletzung erlangt. Weist ein Betroffener den Hostprovider auf eine Verletzung seines Persönlichkeitsrechts durch den Nutzer eines Blogs hin, kann der Hostprovider als Störer verpflichtet sein, zukünftig derartige Verletzungen zu verhindern.

Die Rechtsverletzung ergab sich vorliegend aus einer Abwägung des Persönlichkeitsrechts und dem Recht des Providers auf Meinungs- und Medienfreiheit. Das Gericht hob hervor, dass ein Tätigwerden des Hostproviders nur veranlasst ist, wenn der Hinweis so konkret ist, dass der Rechtsverstoß auf der Grundlage der Behauptungen des Betroffenen ohne Schwierigkeiten bejaht werden kann. Der Erfahrungsbericht wurde als Tatsachenbehauptung qualifiziert. An sich ließ sich der Inhalt des Erfahrungsberichts zwar als Tatbestand auffassen, dem sowohl Tatsachen als auch Wertungselemente („Vorsicht!“, „Fuscher!“, „schlimmer [...] verunstalten", "furchtbar") innewohnen. Schlussendlich war die Darstellung schwerpunktmäßig insgesamt wie eine Tatsachenbehauptung zu behandeln. Der Eintrag fiel nämlich im Tatsächlichen sehr detailliert aus. Es soll eine Operation gegeben haben, in deren Folge der „Patient" Schäden am Gesäß und an den Armen erlitten haben will. Er will Nachbehandlungen in Form von Korrekturoperationen über sich ergehen lassen haben. Und er soll für diese Nachbehandlungen auch „investiert“ haben. Nichts soll jetzt mehr "helfen“.

Es war rechtsunerheblich, ob die Tatsachenbehauptung richtig oder falsch ist. Denn den Hostprovider traf schon deswegen die Haftung, weil er es unterlassen 
hatte, den gesamten Sachverhalt zu recherchieren und zu evaluieren, unter Berücksichtigung einer allfälligen Stellungnahme des für den Eintrag Verantwortlichen. Der Arzt hatte ausreichend darauf hingewiesen, dass der beanstandete Eintrag verleumderisch sei und er diesen gelöscht sehen will. Mit „verleumderisch“ hat der Arzt das zum Ausdruck gebracht, was Tatbestandsvoraussetzung des strafrechtlichen Verleumdungstatbestands ist: Im Eintrag in Beziehung auf den Arzt waren unwahre Tatsachen enthalten, die denselben verächtlich zu machen oder in der öffentlichen Meinung herabzuwürdigen oder dessen Kredit zu gefährden geeignet sind. Die so formulierte Beanstandung des Arztes genügte dafür, dass Prüfungspflichten des Hostproviders ausgelöst werden. Es war auch nicht rechtlich relevant für den Anspruch, dass der Hostprovider den Eintrag doch noch gelöscht hatte. Dies geschah nach Ansicht der Richter zu spät.

\section{Internet-Blog}

\section{$\nabla$}

Die zuvor dargestellten Entscheidungen haben gemeinsam, dass sie die Ansprüche eines Arztes gegenüber demjenigen behandeln, der den relevanten Inhalt unmittelbar im Internet bereithält. Anders ist dies bei einem Linksetzer, der nur mittelbar Zugriff auf die digitalisierten Informationen bietet.

\section{Hintergrund}

Hintergrund des Urteils des Landgerichts Hamburg [4] im Rahmen einer bürgerlich-rechtlichen Rechtsstreitigkeit war, dass ein Arzt in seinen zwei Arztpraxen eine Eigenblutzytokine-Behandlung von Patienten mit Krebsleiden anbot. Auf einer Webseite im Internet wurde in einem „Blog zum Medienrecht“ wie folgt berichtet:

„Dr. K. - Klagen bis der Arzt kommt (1) Zu den zähesten Dauerkunden der Medienjurisprudenz darf sich der Krebsarzt Dr. K. zählen, der heute mal wieder Termin vor dem LG Hamburg hat, diesmal gegen einen Fernsehsender [Anm. der Redaktion] wegen dem obigen Fernsehbeitrag [Anm. der Redaktion]. Bei jeder mir bekannten K.Klage argumentiert der gute Mann stets mit einem vierseitigen Gutachten einer Klinik [Anm. der Redaktion] von 1999, das angeblich die Wirksamkeit seiner Heilkünste belege. Dabei vergisst der erfahrene Kläger regelmäßig zu erwähnen, dass der
,Gutachter' längst mit Bausch und Bogen aus dieser Klinik [Anm. der Redaktion] geflogen ist. Der Klinik [Anm. der Redaktion] reichte schließlich K. Hausieren mit ihrem guten Namen: Letztes Jahr verklagte sie K. erfolgreich auf Unterlassung. Das Urteil wurde kürzlich durch das OLG München bestätigt. Wir werden uns in absehbarer Zeit noch öfters mit Dr. K. zu beschäftigen haben."

Unter der Überschrift „Dr. K. - Klagen bis der Arzt kommt (1)“" konnte durch Anklicken eines Hyperlinks ein vom Fernsehsender im Rahmen einer Fernsehsendung ausgestrahlter Fernsehbeitrag abgespielt werden. Der Beitrag befand sich auf einem externen Server des Videokanals. Im Beitrag wurden die Symbole der Fernsehsenders und des Videokanals eingeblendet. Der Fernsehbeitrag beinhaltete heimlich erstellte Aufnahmen aus den Praxisräumen.

\section{Erwägungen}

Das Landgericht Hamburg entschied, dass der Unterlassungsanspruch des Arztes gemäß §1004 Abs.1 S.2 BGB analog begründet war. Der Fernsehbeitrag verletzte den Kläger in seinem allgemeinen Persönlichkeitsrecht. Es entstand der ehrenrührige Eindruck, dass der Arzt in seiner Arztpraxis Eigenblutpräparate an $\mathrm{Pa}$ tienten aushändige. Für den streitgegenständlichen geltend gemachten Eindruck in Bezug auf die Arztpraxis war unerheblich, ob in der anderen Arztpraxis Eigenblutpräparate ausgehändigt wurden. Ferner entstand in dem Fernsehbeitrag der ehrenrührige Eindruck, die Existenz eines Gutachtens zur Wirksamkeit der Therapien liege nicht vor. Dies war im Gegenteil tatsächlich der Fall. Auch verletzte die Verbreitung der heimlich erstellten Aufnahmen aus den Praxisräumen den Arzt in seinem allgemeinen Persönlichkeitsrecht. Die Aufnahmen waren auf rechtswidrige Art und Weise unter Verletzung des Hausrechts des Arztes zu Stande gekommen. Neben den Grundsatz, dass ein Hausrechtsinhaber es nicht hinnehmen muss, dass gegen seinen Willen in dem seinem Hausrecht unterliegenden Bereich Filmaufnahmen und Fotoaufnahmen gefertigt werden, trat die Besonderheit, dass es sich bei der Arztpraxis für Krebskranke um einen besonders geschützten Raum handelte. Dieser Umstand führte dazu, dass das Hinwegsetzen über das Hausrecht des Inhabers zugleich eine Verletzung des allgemeinen Persönlichkeitsrechts bedingte. Der Arzt wurde nicht um Erlaubnis gebeten, innerhalb seiner Praxisräume Aufnahmen erstellen zu dürfen. Vielmehr gab man sich als Angehöriger eines Krebskranken aus, um so einen Termin in der Praxis zu erhalten und dann mit versteckter Kamera Aufnahmen zu erstellen und eine Patientin zu filmen. Die Ausstrahlung der durch Täuschung entstandenen Aufnahmen erwies sich ebenfalls als rechtswidrig. Es wurden keine Missstände von erheblichem Gewicht aufgedeckt, an deren Aufdeckung ein überragendes öffentliches Interesse bestand. Denn die Abgabe von Eigenblutpräparaten in der Arztpraxis galt - wie bereits ausgeführt - als prozessual nicht wahr. Der Betreiber des Internet-Blogs haftete als Verbreiter des Fernsehbeitrags. Indem er einen Hyperlink auf eine sich auf einem externen Server befindliche Fernsehberichterstattung über den Arzt im Rahmen einer eigenen redaktionellen Berichterstattung über den Arzt integrierte, verbreitete er diesen Fernsehbeitrag. Der Blog-Verantwortliche verletzte auch seine Prüfpflichten. Besteht konkreter Anlass, an der Wahrheit der in einem verbreiteten Beitrag enthaltenen Tatsachenbehauptungen $\mathrm{zu}$ zweifeln, so muss jedenfalls vor einer weiteren Verbreitung bei dem Betroffenen nachgefragt werden. Indem der BlogVerantwortliche dieses und auch jede anderweitige Prüfung des Beitrags auf seine Rechtmäßigkeit unterließ, hat er die ihm nach den konkreten Umständen des Einzelfalls aufzuerlegenden Prüfungspflichten verletzt. Diese Prüfpflichten gelten insbesondere, wenn gleichzeitig jegliche Distanzierung von der verbreiteten Berichterstattung unterbleibt.

\section{Fazit}

$\nabla$

In faktischer Hinsicht ist aufzuzeigen, dass eine wissenschaftliche Untersuchung der Hochschule Worms von anderen Bewertungsportalen (z.B. Hotelbewertung) hervorbrachte, dass mit zunehmendem Informationsumfang die Glaubhaftigkeit der Bewertung zunahm [5]. Es ist nicht ersichtlich, warum dies nicht auch für Arzt-Bewertungen Geltung beanspruchen sollte. Arzt-Bewertungsportale haben grundsätzlich einen höheren Rang bei Suchmaschinen als die einzelnen Arztpraxen. Dies bedeutet, dass die Sichtbarkeit einer Arztpraxis oder eines Arztes im Internet zunimmt, sobald Arzt und Arztpraxis auf einem Bewertungsportal präsent sind. In der Summe be- 
deutet dies, dass auch umfangreiche, negative Kommentare über einen Mediziner im Ranking einer Suchmaschine stets obere Plätze in der Trefferliste einnehmen und somit die Wahl eines Patienten im Hinblick auf einen Behandelnden erheblich beeinflussen werden. Es empfiehlt sich daher für Ärzte in regelmäßigen Intervallen bei Arzt-Bewertungsportalen, die nicht nur eine Benotung, sondern auch kommentierenden Text zulassen (z.B. jameda, Google-Erfahrungsbericht), eine Untersuchung auf negative Erfahrungsberichte vorzunehmen. Wird ein nachteiliger Bericht über Erfahrungen mit der Arztpraxis oder dem Behandelnden festgestellt, sollte der Arzt im Fortgang zunächst einmal prüfen, ob überhaupt ein entsprechender Patient (z.B. in dem Quartal, mit der Therapie) in Behandlung war.

Aus dem rechtlichen Blickwinkel heraus ist die Ausdifferenzierung zwischen Tatsachenbehauptung und Meinung hervorzuheben. Als Heuristik für diese Unterscheidung prüft der Arzt, ob die Äußerung den Vorwurf vorgeblicher Behandlungsfehler enthält, die in einem Arzthaftungsprozess einem Sachverständigengutachten zugänglich wären. Dies würde für eine Tatsachenbehauptung sprechen. Ferner ist hervorzuheben, dass im einstweiligen Rechtsschutz teilweise eine Monatsfrist (ab Kenntnisnahme des Verstoßes) gilt. Bei deren Versäumnis kann der Arzt den Unterlassungsanspruch wegen unrichtiger Behauptungen nicht mehr mit einer einstweiligen Verfügung geltend machen, sondern nur noch im teils langwierigen Klageverfahren. Die zuvor skizzierten zivilrechtlichen Urteile haben eine strafrechtliche Kehrseite. Aus Sicht des Arztes kann dieser für sich die strafrechtlichen Ehrverletzungsdelikte ( $\$ \S 185$ ff. StGB) bei nicht berechtigtem Vorwurf von Behandlungsfehlern „beanspruchen“. Neben dem Strafrechtstatbestand der Beleidigung kommen Verleumdung ( $\$ 187$ StGB) und üble Nachrede ( $\$ 186$ StGB) in Betracht. Grundsätzlich handelt es sich dabei um Delikte, für die der Arzt einen Strafantrag stellen muss. Aber auch aus dem Aspekt, dass der Arzt als Straftäter in Betracht kommt, sollte er sondieren, ob er gegen eine unzutreffende Behauptung eines Behandlungsfehlers vorgeht. Jede ärztliche Behandlung stellt eine tatbestandliche Körperverletzung dar im Sinne der $\$ \S 223$ ff. StGB. Brisant wird es, wenn der Internetbericht den
Tatverdacht einer schweren Körperverletzung weckt. Dies war im Fall des Landgerichts Berlin [3] klar denkbar, weil nach der Berichterstattung die Verunstaltungen an mehreren Stellen des Körpers nicht mehr mit Schönheitsoperationen zu beseitigen und daher dauerhaft waren. Diesen Fall bewehrt das Strafrecht nicht mehr mit einer Geldstrafe, sondern mit einer Mindestfreiheitsstrafe von einem Jahr bis zu zehn Jahren. Zugleich und auf diese Gefahr ist hinzuweisen wird diese Straftat nicht mehr nur auf Antrag des Patienten, sondern von Amts wegen verfolgt. Nehmen die Ermittlungsbehörden z.B. auf anonymen Hinweis oder bei Sichtung des Internets einen derartigen Sachverhalt zur Kenntnis, wird - unabhängig von der Berechtigung des Patientenberichts - ein Ermittlungsverfahren gegen den Arzt eingeleitet. Ein Ermittlungsverfahren kann auch bei einfachen Körperverletzungen initiiert werden, wenn ein besonderes öffentliches Interesse besteht. Da es sich bei Patientenberichten über (angeblich) misslungene Behandlungen um strafrechtliche Verfehlungen im Kontext mit der Ausübung der Heilkunde handelt, kann dies auch die „Unwürdigkeit“ als tatbestandliche Voraussetzung für die Aufhebung der Approbation erfüllen. Zuletzt bestätigte das VG Magdeburg den Widerruf der Approbation u.a. aufgrund von Behandlungsfehlern [6]. Für die Approbation sind ebenso angebliche Unregelmäßigkeiten bei der Abrechnung problematisch. Wenn das Landgericht Nürnberg-Fürth [1] darauf hinwies, dass in einem zivilrechtlichen Haftungsprozess geprüft werden könnte, ob der Zahnarzt ordnungsgemäß abrechnete, dann kann dies als strafrechtliche Anlage den Vorwurf des Betrugs gemäß $§ 263$ StGB beinhalten und zu einer Prüfung Anlass geben. Mehren sich derartige Kommentare in puncto Abrechnung gegenüber Patienten, kommt ein besonders schwerer Fall des Betrugs gem. §263 Abs.3 Nr.1 StGB (gewerbsmäßig) in Betracht. Auch diese Problematik können kritische Kommentare in sich tragen.

Das von den Ärzten in den Entscheidungen praktizierte zivilrechtliche Vorgehen kann daher auch strafrechtliche Ermittlungen und Approbationsverfahren abwehren. Ein „Monitoring“ von Internetinformationen über den Arzt kann aus diesem Grunde geboten sein. Ob es sich der Arzt leisten kann, die über ihn bereit ge- haltenen Informationen im Internet einfach zu ignorieren, dürfte angesichts der vorherigen Darstellung zu verneinen sein.

Autorenerklärung: Der Autor erklärt, dass er keine finanziellen Verbindungen mit einer Firma hat, deren Produkt in dem Artikel eine wichtige Rolle spielt (oder mit einer Firma, die ein Konkurrenzprodukt vertreibt).

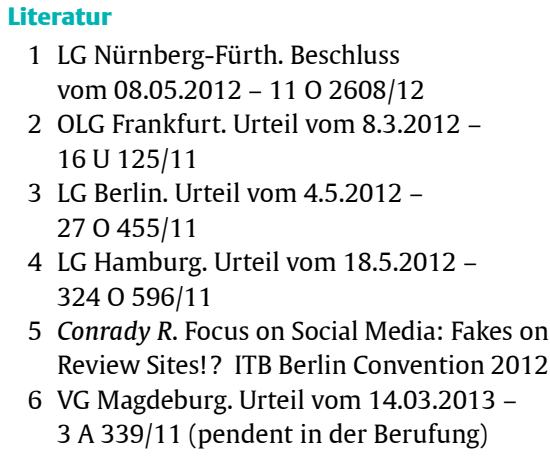

3 LG Berlin. Urteil vom 4.5.2012 $270455 / 11$

4 LG Hamburg. Urteil vom 18.5.2012 $3240596 / 11$

5 Conrady R. Focus on Social Media: Fakes on Review Sites!? ITB Berlin Convention 2012

6 VG Magdeburg. Urteil vom 14.03.2013 3 A 339/11 (pendent in der Berufung) 